\title{
Study on Improving the Stability of Blueberry Anthocyanins
}

\author{
Qing $\mathrm{Xu}^{1}$, Yuanjing Zhou ${ }^{2,{ }^{*}}$, Daibo Wang ${ }^{1}$, Bingjing $\mathrm{Li}^{1}$, Liping Luo ${ }^{1}$ and \\ Guilong Gao, ${ }^{1, *}$ \\ ${ }^{1}$ Institute of Biology, Guizhou Academy of Sciences, Guiyang 550009, China \\ ${ }^{2}$ Institute of Analysis and Testing, Guizhou Academy of Sciences, Guiyang 550002, China \\ *email: xqhaoy@163.com, zhouyuanjing-8006@163.com
}

Keywords: Blueberry anthocyanins, stability, acylation, nanoparticles, microencapsualtion, copigmentation

Abstract. Blueberry anthocyanins are the major active ingredients of blueberry with a variety of biological activities and have widespread applications in the fields of drugs, cosmetics, food additives and so on. However, the instability of blueberry anthocyanins is a big obstacle for their usage. Improving the stability of pigment is the key to promote its development and application. The study on improving the stability of blueberry anthocyanins including physical and chemical methods has been done in recent year and this article reviewed the advance. This work would provide useful references for further application and industrial production of blueberry anthocyanins, as well as may help promote application of anthocyanins from most plant materials.

\section{Introduction}

Anthocyanins, as highly colored substances found in plants, are possible viable colorants for using in food, nutraceutical, and pharmaceutical preparations with a variety of biological effect helping to our human health. However, natural pigment exists some defects, especially its poor stability, which is affected by $\mathrm{pH}$, oxidation, light, temperature and so on. In the process of processing and circulation, it is vulnerable to external influence and can be easily degraded. Therefore, improving the stability of pigment is the key to promote the development and application of natural pigment ${ }^{[1-3]}$. Bluebery (or bilberry), also known as Vaccinium spp., is rich in anthocyanidins with a variety of biological activities $^{[2]}$, and be considered one of the best potential sources of antioxidants in the diet. However, the instability of blueberry anthocyanins is a big obstacle for their usage. The study on stability of blueberry anthocyanins including physical and chemical methods has been done in recent year and this article reviewed the advance.

\section{Stability of blueberry anthocyanins}

Numerous studies have shown that the stability of blueberry anthocyanins is poor and vulnerable to external factor, such as its own molecular structure, $\mathrm{pH}$, temperature, light and others ${ }^{[4-8]}$.
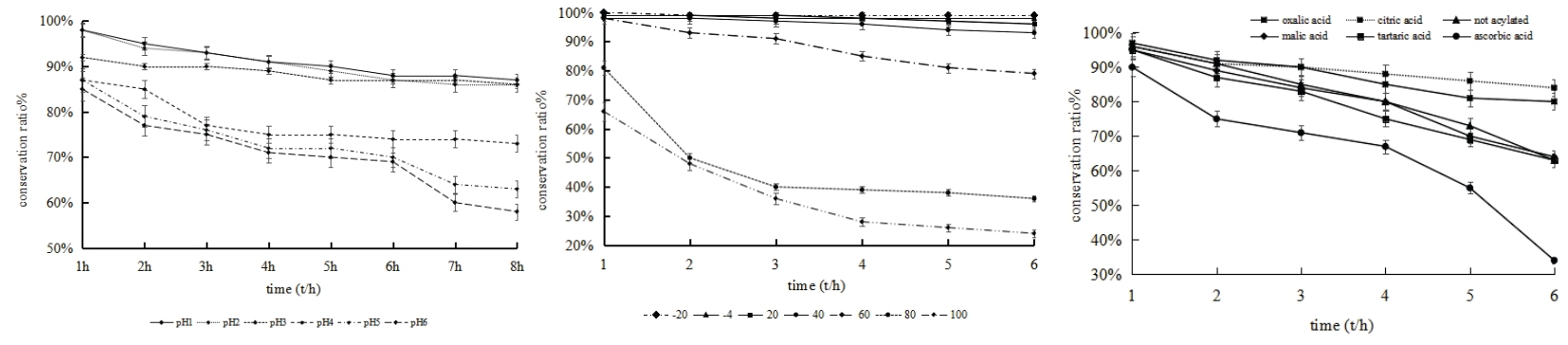

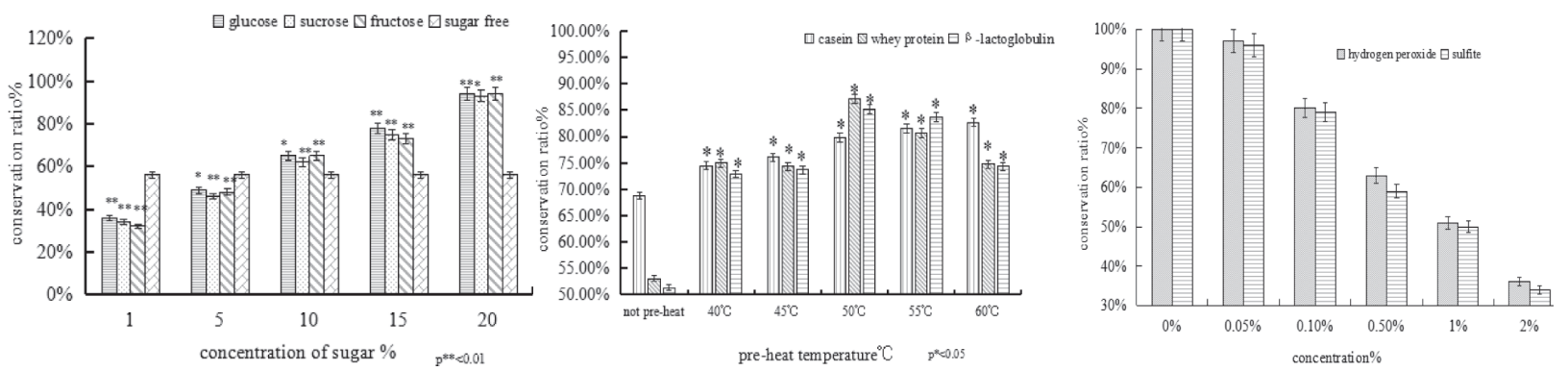

Figure 1 The stability of anthocyanins from blueberry

As shown in Figure 1, the anthocyanins extracted from blueberry fruits were significantly influenced by organic acid $(\mathrm{pH} \leq 3)$, and low temperature. High concentrations of glucose, fructose, sucrose, $60^{\circ} \mathrm{C}$ preheated casein, $50^{\circ} \mathrm{C}$ preheated whey protein and lactoglobulin, citric acid, oxalic acid could protect the stability of anthocyanins ${ }^{[6]}$.

Reque'study showed that there were significant losses of anthocyanins both in the frozen blueberry fruits $\left(-18^{\circ} \mathrm{C}\right.$ for 6 months, $\left.59 \%\right)$ and refrigerated blueberry juice $\left(4^{\circ} \mathrm{C}\right.$ for $\left.10 \mathrm{~d}, 83 \%\right)$. Whereas the anthocyanins were degraded, possibly due to oxidations and/or condensation reactions with other phenolic compounds ${ }^{[7]}$. Iwona's result demonstrated probiotic cultures had significant impact on the stability of blueberry anthocyanins for production of yoghurt, and an appropriate selection of culture was recommended ${ }^{[8]}$.

Studies have shown that anthocyanins usually have better in vitro bioactivity than in vivo bioactivity. Liu's result demonstrated that gastric digestion did not significant affect blueberry anthocyanins in terms of composition and antioxidative activity. However, approximately $42 \%$ of the total anthocyanin and $29 \%$ of the antioxidative activity were lost during intestinal digestion. Structural analysis indicated that fewer free hydroxyl groups and more methoxy groups in the B-ring improved anthocyanin stability. The absorption trials demonstrated that more hydrophobic anthocyanins had better absorption efficiency than more hydrophilic anthocyanins. This might be due to physiochemical degradation during gastrointestinal digestion and their poor bioavailability in vivo studies ${ }^{[9]}$.

\section{Study on improving the stability of blueberry anthocyanins}

Physical method. The physical method to improve the stability of blueberry anthocyanins included copigmentation, carrier adsorption, microencapsualtion, and other methods.

Copigmentation. By copigmentation, the stability of anthocyanins in blueberry extract and blueberry juice could be improved. For example, Jamei et al. investigated the copigments, including tannic, caffeic, benzoic, and coumaric acids, in various concentrations on the stability of blueberry anthocyanin, and concluded that among the tested co-pigments, caffeic acid was the best copigment, while benzoic acid was the weakest ${ }^{[10]}$. Pan et al. studied the flavonoid on the stability of blueberry juice, and indicated that the flavonoid $\mathrm{C}$-glycoside extracts from pigeon pea leaves showed dramatic effect on the color and anthocyanins stability of blueberry juice ${ }^{[11]}$.

Carrier adsorption. Encapsulated with protein molecules, anthocyanins could be protected owing to the slowing down of the oxidation process. Chen et al. investigated the oxidation stability of anthocyanins nanoparticles formed by self-assembly the anthocyanins and bovine serum albumin (BSA), and concluded that BSA nanocarriers could improve the stability of anthocyanin under neutral conditions ${ }^{[12]}$. They also evaluated the stability of Cyanidin-3-glucoside (Cy-3-glu) particles that adsorbed by BSA. Their results indicated that Cy-3-glu nanoparticles were improved significantly in the stability of temperature, illumination and strong oxidizer reducer comparing with unbound anthocyanin, and Vitamin C (VC), glucose and sucrose could also improve the stability of adsorbed Cy-3-gluby BSA ${ }^{[13]}$.

Microencapsualtion. In order to make them full applications, microencapsulation technology was used to solve the instability of blueberry anthocyanins ${ }^{[14]}$. Li et al. studied the microencapsulation of blueberry anthocyanins by using ethyl cellulose, mixture of soybean protein isolation-maltodextrin, 
and mixture of $\beta$-cyclodextrin-maltodextrinn-arabicgum, separately as the wall material, and the temperature and light stability of microencapsulation of blueberry anthocyanins was improved ${ }^{[15,16]}$.

Other methods. In addition to the methods mentioned above, other methods to stabilizing blueberry anthocyanins had acidification $(\mathrm{pH} 2.1)$ and refrigeration $\left(4^{\circ} \mathrm{C}\right)$ for blueberry juice ${ }^{[17]}$, high temperature short time sterilization $\left(121^{\circ} \mathrm{C}, 10 \mathrm{~s}\right)$ and stored with the full-label packaging for blueberry beverage $^{[18]}$.

Chemical method. Some studies reported that the acylation of blueberry anthocyanins had high stability ${ }^{[19,20]}$. We acylated blueberry anthocyanins by using acetic acid, oxalic acid, propionic acid, succinic acid, heptane acid, and lauric acid, and investigated the effect of different storing environment, light and heat on the stability of blueberry anthocyanins ${ }^{[21,22]}$. As shown in Figure 2, the stabilities of acylated anthocyanins under light and heat were significantly increased being compared with no acylated anthocyanins. And the stability of acylated blueberry anthocyanin increased with the increase of carbon chain of aliphatic carbonyl acid. When the number of carbon was the same, binary carboxylic acids were more stable on temperature and light than monocarboxylic acids.
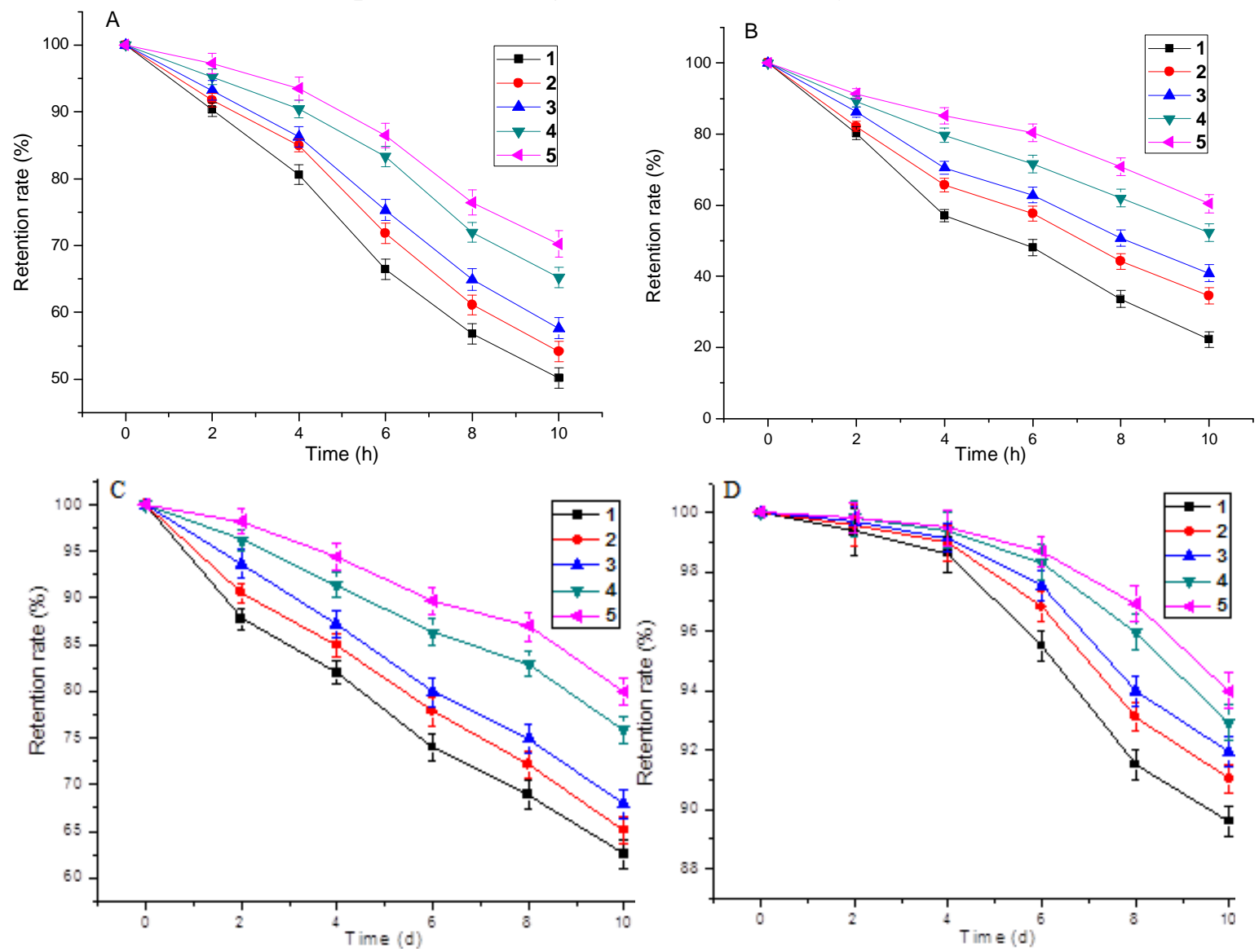

Figure 2 The stability of blueberry anthocyanin and acylated blueberry anthocyanin. A: temperature at $80^{\circ} \mathrm{C}$; B: temperature at $100^{\circ} \mathrm{C}$; C: indoor natural light; $\mathrm{D}$ : indoor avoiding light. 1: blueberry anthocyanin; 2: acetic acid acylated blueberry anthocyanin; 3: oxalic acid acylated blueberry anthocyanin; 4: heptane acid acylated blueberry anthocyanin; 5: lauric acid acylated blueberry anthocyanin.

\section{Conclusions}

Blueberry anthocyanins belong to flavonoids with various biological activities. They are widely applied in food and medicine fields, but the properties of anthocyanins are very unstable, which are easily interfered by external factors. Therefore, we should strengthen the study of the stability of blueberry anthocyanin, which has made some progress, yet we should continue to strengthen the study on anthocyanin stabilization and application so that the anthocyanins can get more practical applications and development. 


\section{Acknowledgements}

This work was financially supported by the Youth Fund Project of Guizhou Academy of Sciences, Guizhou province, China (J [2016]20), Science and Technology Project of Guizhou Province, China (JZ [2015]2006, Z [2015]6013-4, [2016]1139, [2017]01), and National Natural Science Foundation (81560603).

\section{References}

[1] X.X. Zeng, L. Lei, G.H. Zhao and F.Y. Ye: Food science, in press.

[2] Y. Zuo and F. Tian: Food and grease Vol. 27 (2014), p. 1

[3] X.Y. Tian, J.H Deng, J.J Wei and S. Liu: Academic Periodical of Farm Products Processing, (2012), p.126

[4] H.G. Yan, W.H. Zhang and A.E. Ding: Science and Technology of Food Industry Vol. 34 (2013), p. 119

[5] Q. Xu, Y.J. Zhou, Y.X. Wu, et al. Biotechnology \& Medical Science, (2016), p.104

[6] Q.Y. Sun, S. Ren, B.J. Lu and J. Zhang: Acta Nutrimenta Sinica Vol. 39 (2017), p. 400

[7] P.M. Reque, R. S. Steffens, A. Jablonski, et al. Subtropical plant science Vol. 33 (2014), p. 111

[8] Ś. Iwona, Z. Małgorzata, M. Marta and Z. Dorota: LWT-Food Sci. Technol. Vol.49 (2012), p. 208

[9] Y.X. Liu, D. Zhang, Y.P. Wu, et al. Int. J. Food Sci. Nutr. Vol. 65 (2014), p. 440

[10] R. Jamei and F. Babaloo: Int. J. Food Prop. Vol. 20 (2017), p. 2128

[11] Y.Z. Pan, Y. Guan, Z.F. Wei, et al. Ind. Crop. Prod. Vol. 58 (2014), p.142

[12] J. Chen, X.Y. Tao, M. Zhang, et al. J. Sci. Food Agr. Vol. 94 (2014), p.1781

[13] X.L. Lin, C.N. Liu, X. Zhang and J Chen: Journal of Anhui Agricultural Sciences (2015), p. 151

[14] B. He, J. Liang, L.S. Wu and X.L Gao: Chinese Agricultural Science Bulletin Vol. 31 (2015), p. 127

[15] Y.C. Li, Z.L. Ma, et al. Science and Technology of Food Industry Vol. 30 (2009), p. 250

[16] Y.C. Li, C.M. Lv, X.J. Meng, et al. Food and fermentation industries, Vol.36 (2010) , p. 71

[17] L.R. Howard, C. Brownmiller, A. Mauromoustakos and R.L. Prior: Journal of Berry Research Vol.6 (2016), p. 189

[18] Y. Jia and Z.H. Hu: Journal of Nuclear Agricultural Sciences Vol.30 (2016), p. 941

[19] Y.C. Li, B.X. Li, C.M. LV and X.J. Meng: Science and Technology of Food Industry Vol. 33 (2012), p. 212

[20] L.Y. Zhao, L.N. Li, R.M. Shen, et al. Science and Technology of Food Industry, Vol. 35 (2014), p. 299

[21] Q. Xu, Y.J. Zhou, L.P. Luo, et al. Advances Engineering Research, Vol. 143 (2017), p. 907

[22] Q. Xu, Y.J. Zhou, Liping Luo, et al. Advances Material Energy \& Enviromental Engineering, (2017), p. 378 